\title{
What can we salvage from care.data?
}

\author{
Fiona Godlee editor in chief
}

The BMJ

By now, if all had gone to plan, England would be reaping the early benefits of a national database of patients' medical records spanning primary and secondary care. Patients would have the comfort of knowing that their records could soon be accessed wherever they were treated; the ability to monitor outcomes might already be suggesting better ways of doing things; and researchers might be starting to interrogate anonymised datasets to generate and test hypotheses.

But all did not go to plan. NHS England's care.data programme failed to win the public's trust and lost the battle for doctors' support. Two reports have now condemned the scheme, and last week the government decided to scrap it (doi:10.1136/bmj. i3804).

This failure is hugely disappointing. The NHS is, on the face of it, the ideal setting for safe and fruitful sharing of data, with potentially enormous benefits for patients both current and future. In her report Fiona Caldicott, the national data guardian, does not hide her frustration that her earlier advice in two previous reports was not heeded. Increasingly frequent data breaches confirmed growing fears among GPs and sapped public confidence. Those in charge of the project failed to adequately explain the benefits of data sharing. They also allowed the toxic possibility that personal data might be accessed by commercial companies. The decision to make patients actively opt out proved an additional misjudgment.

So it's back to the drawing board. As Tjeerd-Pieter van Staa and colleagues explain, public trust will be the key to success of any future "big health data" project (doi:10.1136/bmj.i3636). But England can learn from other countries. Scotland, Wales, Canada, the US, and Norway all offer models to emulate, they say.

The Caldicott report sets out what is needed, including better technology standards, proper marketing of the benefits, an easy opt-out procedure, and a "dynamic consent" process. The Care Quality Commission adds further stringent recommendations. "The NHS cannot afford to wait any longer for better data," says Nicola Perrin in her editorial (doi:10.1136/bmj.i3852). But perhaps in redrawing our plans we should leapfrog outdated views on ownership of information. Having failed with a centralised approach, could now be the time for the NHS to give patients control of their own medical records? Several experiments and pilot projects show that this can work (doi: 10.1136/bmj.i722, doi: 10.1136/bmj.g7785).

If the banking industry can manage this, why can't health? 